\title{
THE MAGNETIZATION OF HEUSLER ALLOYS AS A FUNCTION OF THE TEMPERATURE AND CALCULATION OF THE INTRINSIC MAGNETIC FIELD.
}

\author{
By Percy Wilcox Gumaer.
}

$\mathrm{T}^{\mathrm{H}}$ E magnetic alloys of manganese are composed of metals which ordinarily are non-magnetic. Manganese itself is not only nonmagnetic, but a small per cent of it will reduce the magnetic properties of iron. It is probable that the explanation of these magnetic alloys will add considerable to our understanding of the ultimate nature of magnetism.

Recent developments in the electron theory of magnetism have opened up a means of studying the molecular structure of the alloys.

The present investigation was undertaken with two objects in view, first: to study the effect of temperature upon the saturation value of the intensity of magnetization. Then, to determine, if possible, from the data obtained, the structure of the molecular magnets.

\section{METHOD.}

To determine the saturation value of the intensity of magnetization the method used by Weiss ${ }^{1}$ and Stifler ${ }^{2}$ was chosen. A ballistic galvanometer was connected in series with a helix placed in a strong magnetic field. An ellipsoid of the alloy to be tested was placed in the center of this helix and the deflection of the galvanometer was observed as the ellipsoid was, quickly, pushed out. The deflection of the galvanometer was then compared to that obtained from the current induced in the secondary of a standard helix which was included in the circuit. The intensity of magnetization $I$ can be determined from the relation:

$$
I=\frac{k}{v} \frac{i^{\prime}}{d^{\prime}} d
$$

where $k=$ a constant depending upon the dimensions of the helix and of the standard helix.

$v=$ the volume of the ellipsoid.

$i=$ current in the primary of the standard helix.

$d^{\prime}=$ deflection due to induced current in the standard helix.

$d=$ deflection when ellipsoid is removed from the helix.

1 Archives des Sciences, ser. 4, 29, pp. 204 (I910).

2 Phys. Rev., Vol. 33, p. 268 (I9II). 
The ellipsoid and helix were surrounded by a coil of German silver wire, by means of which the desired temperature was obtained. To measure the temperature of the ellipsoid inside the helix a copper-constantan thermo-couple was used. The hot junction was placed in a hard glass tube $3 \mathrm{~mm}$. in diameter and $26 \mathrm{~cm}$. long. Inside the tube the wires were separated by mica strips, outside by $\mathrm{I} / \mathrm{I} 6$ rubber tubing.

The thermo-couple was calibrated by observing the E.M.F. of the couple when the hot junction was at a known temperature. The temperature of steam and the freezing points of metals were used for the calibration, as follows: $\mathrm{Zn} 419.4^{\circ} \mathrm{C}$., $\mathrm{Cd} 321.0^{\circ} \mathrm{C}$., Sn $231.9^{\circ} \mathrm{C}$., steam $100^{\circ} \mathrm{C}$. Using the method of least squares the constants of the equation $E=a t+b t^{2}+c t^{3}$ were determined and the equation becomes

$$
E=3.747 t+.00375 t^{2}+.00000164 t^{3} .
$$

The galvanometer used was a Leeds \& Northrup silver suspension instrument. It had a resistance of $25.6 \mathrm{ohms}$, a ballistic sensibility of $31.8 \mathrm{~mm}$. per micro-coulomb on open circuit, with a scale distance of 50 $\mathrm{cm}$., and a period of II.2 seconds on open circuit. At a scale distance of 6 meters and the deflection could be read to $0.5 \mathrm{~mm}$.

The ellipsoid was inserted directly into the tube forming the core of the helix. It was moved along by pushing with a small glass rod in one end and with the tube containing the thermo-couple in the other end. By this method the diameter of the helix could be decreased by half, which increased the sensitiveness considerably.

The induction helix was wound upon a thin-walled glass tube $45 \mathrm{~cm}$. long and $0.5 \mathrm{~cm}$. outside diameter. Three layers of number 36 silkcovered copper wire were used. The layers were separated by mica, and each layer was covered with a mixture of water glass and calcined magnesia. This mixture became very hard when dry and held the wires firmly in place even at high temperatures.

A hard glass tube long enough to reach to the end of the bore in the magnet was slipped over the helix coil. Thus the possibility of leakage from the heating circuit to the helix coil or lead-in wires was avoided. The heating coil consisted of one layer of 320 turns of number 16 German silver black enameled wire. The winding was done from the middle towards the ends so that the two halves were wound in opposite directions and opposed each other magnetically. As in the induction helix, a mixture of water glass and magnesia was used to hold the wires firmly in place. Since the length of the coil was $30 \mathrm{~cm}$. the temperature gradient in the center was very small.

The induction helix and heating coil were enclosed in a glass tube small 
enough to slide into the bore of the magnet. This tube was filled with calcined magnesia. Further heat insulation between the pole pieces was obtained by enclosing that part of the furnace in a fire clay cylinder filled with shredded asbestos.

The magnetic field was obtained from a large DuBois electromagnet. A hole drilled through the core and pole pieces enabled the ellipsoid to be inserted into the helix. For the air gap used $(6.2 \mathrm{~cm}$.) the strength of the magnetic field in the center of the gap was calibrated in terms of the current in the coils. A magnetic balance was used to measure the strength of the field.

\section{DESCRIPTION OF SPECIMENS.}

The alloys were prepared by melting in a new graphite crucible heated in a gas furnace. The manganese and copper were put in first, and when they were thoroughly fused the aluminum was added. To insure a uniform mixture, the molten alloy was stirred with a graphite rod, and then quickly poured into vertical moulds. Care was taken to pour in a continuous stream so that the oxide formed on the surface would not injure the casting.

The ellipsoids were obtained by grinding the castings with a properly shaped alundum wheel in a Universal Grinder. A projection of the shadow of the ellipsoids showed the cross-section to be fairly accurate.

The dimensions and composition of the two ellipsoids are given as follows:

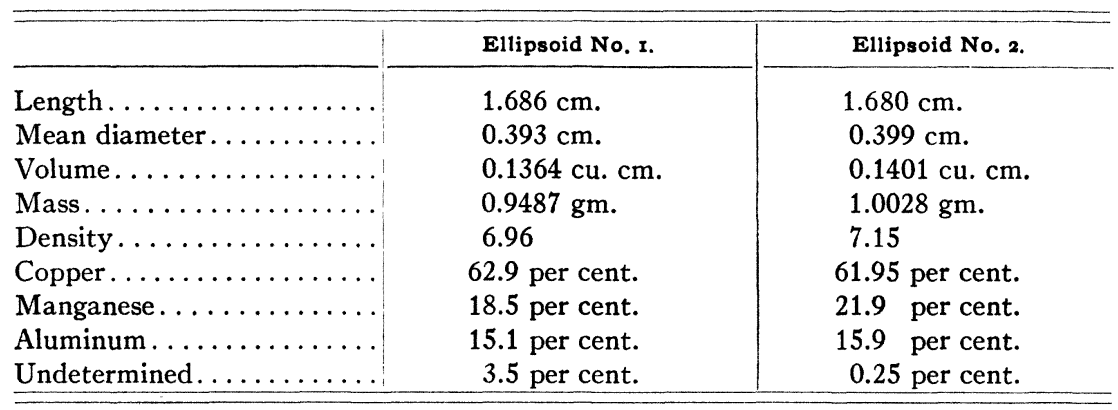

\section{Procedure in Taking Readings.}

After the heating current had been on for a time, sufficient to establish temperature equilibrium, the ellipsoid was inserted into the core of the helix. It was moved along by pushing with the tube containing the thermo-couple from one end and with a glass rod from the other end. A mark on the glass rod indicated when the ellipsoid was in the 
center of the helix. When the temperature had ceased to increase the reading of the thermo-couple was taken, the magnetic field was thrown on and the deflection of the galvanometer was observed as the ellipsoid was quickly pushed out of the helix. A rapid movement of the ellipsoid was obtained by striking the end of the glass rod with a small piece of wood.

The ellipsoid was now replaced in position, allowed to regain its former temperature and the reading repeated. As a rule three readings were taken for each field strength and the intensity of magnetization was calculated from a mean of the three deflections.

At lower temperatures the deflections agreed to within I per cent. but in the neighborhood of the transformation temperature the agreement was not as close. For some of the readings taken above $300^{\circ}$ the maximum deflection was $\mathrm{I} \mathrm{cm}$. at a scale distance of 6 meters. The accuracy in this case was probably about ro per cent.

After each set of readings the galvanometer was calibrated by means of the standard helix. The current in the primary of the helix was read by a Weston milli-ammeter, which had been calibrated by comparison with a standard instrument. The ratio $i^{\prime} ! d^{\prime}$ varied slightly as the temperature increased, due to the increased resistance of the helix coil at higher temperatures. As the heating coil was wound nonmagnetically, it was not necessary to make any correction for it.

Thermo-couple readings were taken just before the ellipsoid was pushed out of the helix. As the end of the tube containing the thermo-couple was left open, and as the couple was

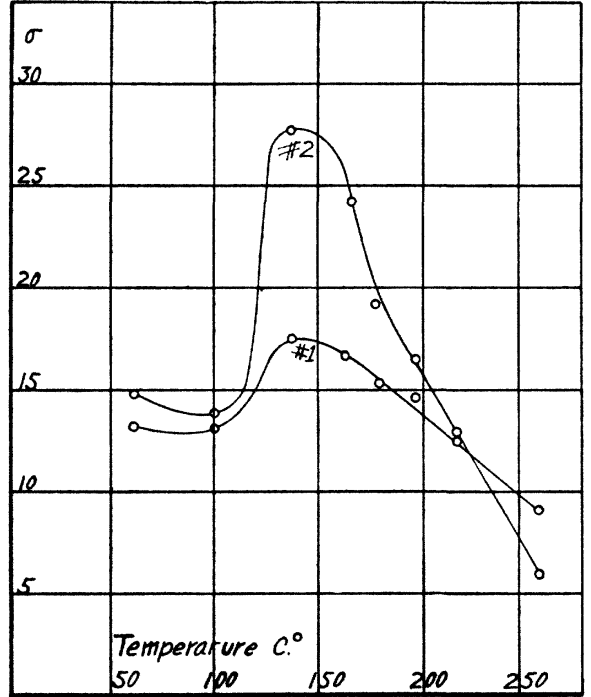

Fig. 1. within a millimeter of the end of the ellipsoid when readings were taken, it is quite probable that the temperature measured corresponded very accurately to the actual temperature of the ellipsoid.

\section{REsults.}

The first set of data obtained is apparently of little theoretical value. The curves (Fig. I) showing the specific intensity of magnetism $\sigma$ as a 
function of the temperature are quite irregular, having a maximum at $140^{\circ}$. Although it is possible that the irregularity of these curves is due to a defect in the apparatus, it is more probable that it is due to the unstable conditions of the alloys. The data were obtained with the alloys in the condition as cast and without previous heat treatment.

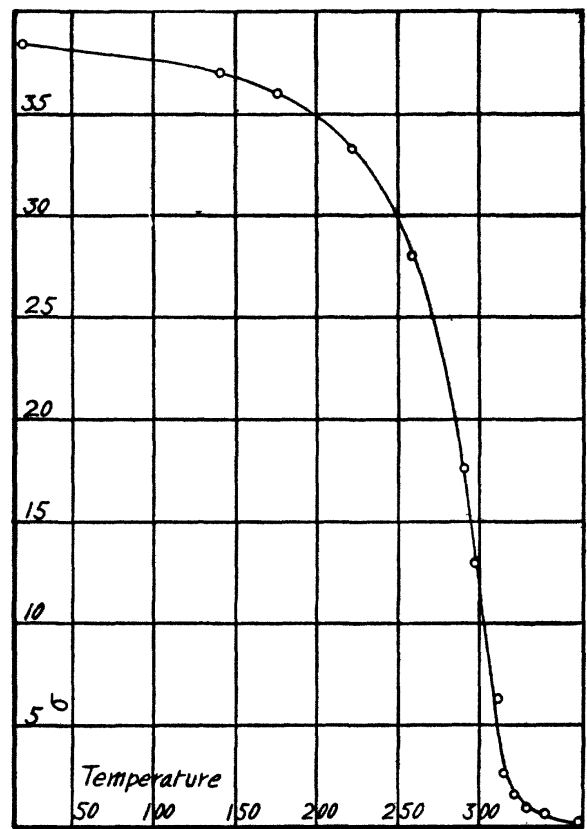

Fig. 2.

Ellipsoid No. 1

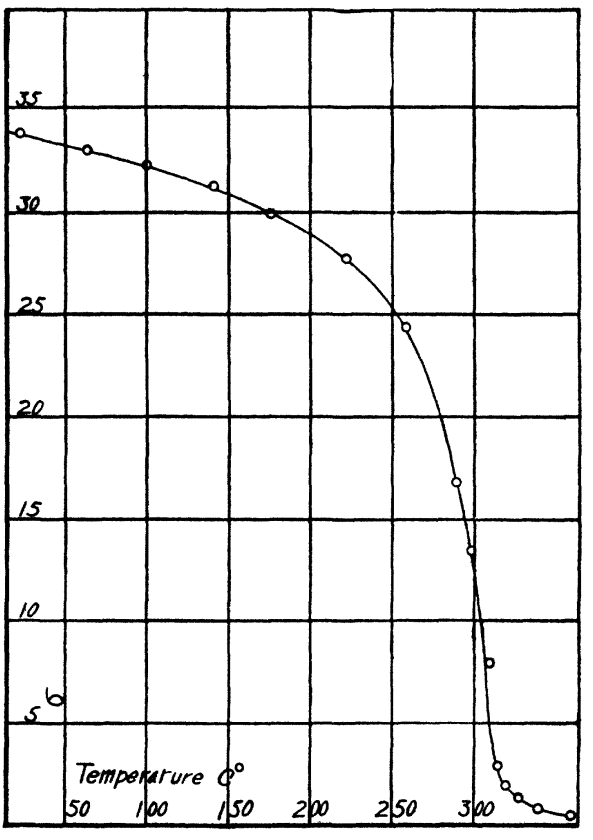

Fig. 3.

Ellipsoid No. 2.

A new helix coil was now built and a series of readings taken at $320^{\circ}$ indicated that the substance had become paramagnetic. Beginning at room temperature, the whole set of data was repeated and very regular curves were obtained, as shown in Figs. 2 and 3 . These curves, showing $\sigma$, the specific intensity of magnetization, as a function of the temperature, are similar to the ones obtained for iron, nickel and cobalt, although they are much flatter at lower temperatures. It is seen from the curves that the temperature of transformation is in the neighborhood of $310^{\circ}$. A theoretical discussion of these curves will be given in a later paragraph.

As the values of $\sigma$ at room temperature were found to be about half of what should be expected from the theoretical calculations, an attempt was made to increase the magnetic intensity by chilling from a temperature near the melting point of the alloy. To do this the ellipsoids were inserted in a quartz tube together with a platinum platinum-rhodium 
thermo-couple, and heated in an electric furnace to $895^{\circ} \mathrm{C}$. They were kept at the temperature for Io minutes and then chilled by plunging the quartz tube into cold water.

The value of the intensity of magnetization was found to have been increased considerably by the chilling. Values of $\sigma$ were obtained, as before, for different values of temperature below the transformation point. The curves are shown in Fig. 4.

In order to be sure of the results the chilling was repeated for ellipsoid No. I and similar values were obtained.

The following table shows a typical set of readings taken at $290^{\circ} \mathrm{C}$. The value of $\sigma$ at each field strength was obtained from the mean of three galvanometer deflections. The temperature was obtained from the mean of the thermo-couple readings. These were not allowed to vary

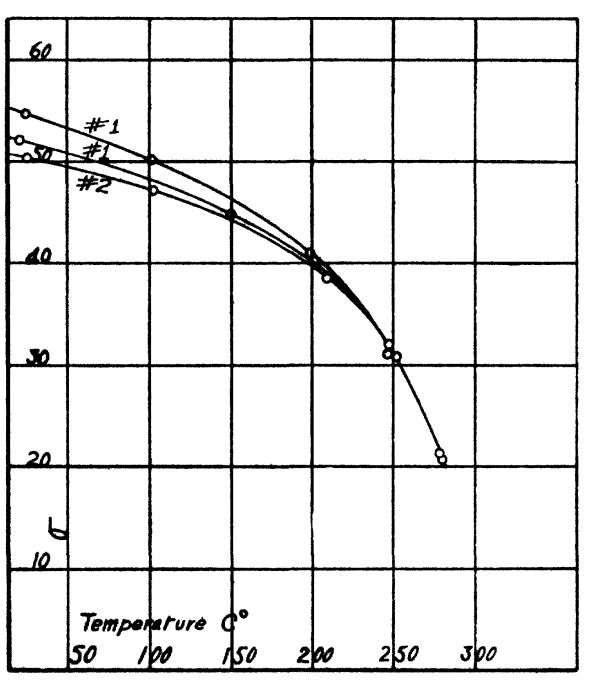

Fig. 4. more than 20 micro-volts, which corresponds to $0.4^{\circ}$.

\begin{tabular}{c|c|c|c|c|c|c|c|c}
\hline$m v$ & $d$ & Mean $d$ & $\sigma$ & $I$ & $L I$ & $I_{m}$ & $H_{0}$ & $H$ \\
\hline 13,670 & 27.5 & & & & & & & \\
13,680 & 27.3 & 27.4 & 15.9 & 114.0 & 85 & 2.0 & 930 & 845 \\
13,675 & 27.5 & & & & & & & \\
13,670 & 27.8 & & & & & & & \\
13,680 & 27.7 & 27.7 & 16.1 & 115.3 & 86 & 3.0 & 1,395 & 1,309 \\
13,670 & 27.7 & & & & & & & \\
& & & & & & & & \\
13,675 & 29.0 & & & & & & & \\
13,665 & 28.8 & 29.0 & 16.8 & 120.5 & 90 & 4.0 & 1,860 & 1,750 \\
13,665 & 29.2 & & & & & & & \\
13,670 & 29.0 & & & & & & & \\
13,670 & 28.7 & 28.9 & 16.75 & 120.0 & 90 & 5.0 & 2,335 & 2,245 \\
13,675 & 29.0 & & & & & & & \\
13,670 & 29.0 & & & & & & & \\
13,670 & 28.8 & 28.9 & 16.75 & 120.0 & 90 & 6.0 & 2,730 & 2,640 \\
13,665 & 28.9 & & & & & & & \\
\hline \hline
\end{tabular}


Typical Set of Data. Taken at $290.9^{\circ}$.

Ellipsoid No. 2.

$$
I=4.157 d, \quad \sigma=0.580 d, \quad L=0.749 \text {. }
$$

$d=$ deflection of the galvanometer,

$m v=$ reading of thermo-couple in microvolts,

$I_{m}=$ current through the magnet,

$H_{0}=$ external field,

$H=$ field inside the ellipsoid $=H_{0}-L I$,

$I=$ intensity of magnetization,

$\sigma=$ specific intensity of magnetization.

Mean value of thermo-couple readings $=13,670 \mathrm{~m} . \mathrm{v}$.

Corresponding temperature $=290.9^{\circ}$.

Deflection due to standard helix $=70.3$.

Current in primary of standard helix $=0.672$ ampere.

\section{Molecular Theory of Magnetism.}

The present theory of magnetism, as developed by Curie, ${ }^{1}$ Weiss, ${ }^{2}$ Langevin ${ }^{3}$ and Kunz, ${ }^{4}$ accounts for the various phenomena by assuming that a magnetic substance is made of small elementary magnets.

In a non-magnetic state these elementary magnets are distributed with their axes pointing equally in all directions. Under the influence of a resultant magnetic field $H$, each elementary magnet is acted upon by a turning force $M H \sin \alpha$, where $M$ is the moment of the elementary magnet and $\alpha$ is the angle between $H$ and the axis of the magnet. The tendency of this couple is to cause the magnets to turn with their axes toward the direction of the existing field. The amount of this rotation depends upon the strength of the field and upon the temperature of the substance.

If there were no thermal agitation of the molecules all the elementary magnets would revolve until their axes coincided with the direction of the existing field. This condition is obtained at absolute zero.

At other temperatures than absolute zero the magnetic energy of the molecules tending to arrange the molecules in the direction of the magnetic field is opposed by the thermal energy. The molecules are continually being deflected by their mutual collisions, and the resultant condition of equilibrium depends upon the ratio of the thermal energy to the magnetic energy.

${ }_{1}^{1}$ Archives des Sciences, ser, 4, 3I, p. 5-19 (I9II).

2 Journal de Physique, 36, p. 66I-690 (1907).

- Annales de Chemie et de Physique, Ser. 5, 8, p. 70-127 (1905).

- Puys. Rev., 30, p. 359-370 (1910). 
Consider a sphere of unit radius within which are a large number of magnetic molecules. When there is no magnetic field acting, the magnets are distributed with their axes pointing equally in all directions. Imagine all these magnets concentrated with their centers at 0 . Under the action of a magnetic field the magnets will be caused to rotate about 0 , and the tendency will be to place their axes in line with the magnetic field. The magnets will no longer have their axes pointing uniformly in all directions, but the magnetic density will be greatest in the direction of $H$. Let us define

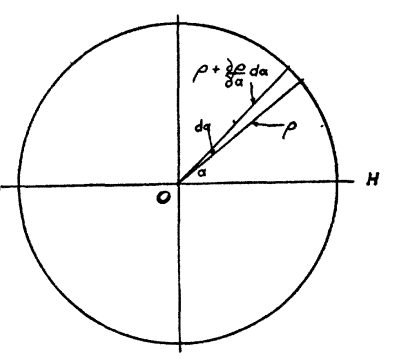

Fig. 5. magnetic density as the number of magnetic axes per unit solid angle or $d n / d \omega$. For abbreviation put $\rho=d n / d \omega$.

Let $\rho$ be the magnetic density at any angle $\alpha$ with the field $H$. Then at angle $\alpha+d \alpha$ the magnetic density will be $\rho+(\partial \rho / \partial \alpha) d \alpha$. The change of magnetic density in moving through angle $d \alpha$ is therefore

$$
\rho-\left(\rho+\frac{\partial \rho}{\partial \alpha} d \alpha\right)=-\frac{\partial \rho}{\partial \alpha} d \alpha .
$$

This change of density depends upon the density $\rho$ at $\alpha$. It is also proportional to a resultant turning force or couple.

If we assume that the molecules of iron, or other ferro-magnetic substance, when in a non-magnetic state are as free to move relatively to each other as the molecules of a gas, then the thermal energy can be deduced from the laws of thermo-dynamics. Since a rotation of the elementary magnet about its own axis has no effect upon its magnetic energy, there remain but two degrees of rotation. Hence, for the molecular magnets, the kinetic energy of heat is equal to $R T$, where $R$ is the universal gas constant and $T$ is the absolute temperature.

In dynamics work/angle $=\mathrm{a}$ couple, hence $R T / d \alpha=\mathrm{a}$ couple due to the thermal energy of the molecules. The magnetic couple acting upon the elementary magnets is $M H \sin \alpha$.

The change of magnetic density varies directly as the magnetic couple and inversely as the couple due to the heat energy; that is, the greater the magnetic couple, the greater will be the change of magnetic density as we pass from $\alpha$ to $\alpha+d \alpha$, and the greater the thermal energy, the smaller will be the change of density. Hence we can put

$$
-\frac{\partial \rho}{\partial \alpha} d \alpha=\rho \frac{M H \sin \alpha}{\frac{R T}{d \alpha}}=\rho \frac{M H \sin \alpha \cdot d \alpha}{R T} .
$$


Then

and

$$
-\frac{\partial \rho}{\partial \alpha}=\rho \frac{M H}{R T} \sin \alpha ;
$$

Integrating

$$
-\frac{\partial \rho}{\rho}=\frac{M H}{R T} \sin \alpha d \alpha .
$$

$$
\log \rho=\frac{M H}{R T} \cos \alpha-\log K
$$

whence,

or

$$
\rho=K e^{\frac{M H}{R T} \cos a},
$$

But

$$
\frac{d n}{d \omega}=K e^{\frac{M H}{R T} \cos a} .
$$

hence

$$
d \omega=2 \pi \sin \alpha d \alpha ;
$$

$$
d n=K e^{\frac{M H}{R T} \cos \alpha} 2 \pi \sin \alpha d \alpha .
$$

Intensity of magnetization may be defined as the product of the number of molecular magnets per unit volume and the moment of the magnets in the direction of the resultant magnetic field. The intensity is a maximum when there is no thermal agitation, so that the molecular magnets are all directed along the field. This condition obtains only at absolute zero.

At other temperatures the intensity due to the magnets whose axes make an angle $\alpha$ with $H$ is

$$
d I=M \cos \alpha d n,
$$

where $M$ is the moment of the molecular magnets, and $I$ is the intensity of magnetization.

Substituting the value of $d n$ from eq. (I), we have

$$
d I=M \cos K e^{\frac{M H}{R T} \cos \alpha} 2 \pi \sin \alpha d \alpha ;
$$

and integrating between limits 0 and $\pi$

Now let

$$
I=\int_{0}^{\pi} M \cos \alpha K e^{\frac{M H}{R T} \cos \alpha} 2 \pi \sin \alpha d \alpha .
$$

$$
\frac{M H}{R T}=a, \quad \cos \alpha=x, \quad-\sin \alpha d \alpha=d x
$$

then, 
But

$$
I=2 \pi M K \int_{+1}^{-1} x e^{a x} d x
$$

$$
\begin{aligned}
\int_{+1}^{-1} x e^{a x} d x & =\frac{a\left(e^{a}+e^{-a}\right)}{a^{2}}-\frac{\left(e^{a}-e^{-a}\right)}{a^{2}} \\
& =2\left(\frac{\cosh a}{a}-\frac{\sinh a}{a^{2}}\right)
\end{aligned}
$$

hence

$$
I=2 \pi M K 2\left(\frac{\cosh a}{a}-\frac{\sinh a}{a^{2}}\right) \text {. }
$$

To evaluate $K$, integrate equation ( 1 ):

$$
\begin{aligned}
n & =2 \pi K \int_{0}^{\pi} e^{a \cos a} \sin \alpha d \alpha \\
& =\frac{4 \pi K}{a} \sinh a ;
\end{aligned}
$$

whence

$$
K=\frac{n a}{4 \pi \sinh a} .
$$

Substituting the value of $K$ in equation (2), we have

$$
\begin{aligned}
I & =2.2 \pi M \frac{n a}{4 \pi \sinh a}\left(\frac{\cosh a}{a}-\frac{\sinh a}{a^{2}}\right) \\
& =M n\left(\frac{\cosh a}{\sinh a}-\frac{\mathrm{I}}{a}\right) .
\end{aligned}
$$

But $M n=I_{m}$; hence,

$$
I=I_{m}\left(\frac{\cosh a}{\sinh a}-\frac{\mathrm{I}}{a}\right)
$$

where

$$
a=\frac{M H}{R T}
$$

Equations (3) and (4) give us an expression for the intensity of magnetization as a function of the temperature.

So far we have considered only the arrangement of the elementary magnets due to the action of an external field. Each magnet, however, has an effect upon the surrounding magnets and the result according to the Weiss theory is a uniform field, proportional to the intensity of magnetization, $I$, and acting in the same direction as $I$. This molecular magnetic field accounts for the great magnetic intensity of iron and 
[VoL. $\mathrm{XXXV}$.

other ferro-magnetic substances in the same way as an internal pressure added to the external pressure accounts for the great density of liquids. The sudden increase of density when a vapor liquefies is due to the fact that an enormous internal pressure is suddenly made effective in addition to the external pressure. Ferro-magnetic substances at high temperatures are but slightly magnetic. As the temperature is slowly decreased a point is reached at which the substances suddenly become very magnetic. This indicates that a strong molecular field has become operative. If $H_{m}$ represents the molecular field and $I$ the intensity of magnetization then $H_{m}=A I$ where $A^{1}$ is a proportionality factor.

The resultant magnetic field within the substance is, consequently, the sum of the external field, $H_{\bullet}$ and the molecular field $H_{m}$, or, $H=H_{\bullet}+H_{m}$.

From equation (4)

whence

$$
T=\frac{M H}{a R}
$$

$$
T=\frac{M\left(H_{\bullet}+H_{m}\right)}{a R} .
$$

The large magnetic intensity of ferro-magnetic substances at ordinary temperatures indicates that the molecular field must be very strong in comparison with the external field. This condition holds up to the temperature at which the substance ceases to be ferro-magnetic. Let $\theta$ be that temperature, then for $T=\theta$, we can neglect $H_{0}$ in comparison to $H_{m}$ and equation (5) becomes

From equation (3)

$$
T=\frac{M A I}{a R}, \quad \text { for } T \leqq \theta .
$$

$$
I=I_{m}\left(\frac{\cosh a}{\sinh a}-\frac{\mathrm{I}}{a}\right) .
$$

The expression $\left(\frac{\cosh a}{\sinh a}-\frac{1}{a}\right)$ can be expanded into the convergent series

$$
\frac{1}{3} a-\frac{2}{90} a^{3}+\frac{4}{45 \cdot 42} a^{5} \cdots .
$$

For very small values of $a$ it is sufficient to consider only the first term of the series, and equation (3) becomes

$$
I=\frac{a I_{m}}{3} \text {. }
$$

1 In order to avoid ambiguity later the symbol $A$ is chosen in preference to $N$, the symbol used by Weiss, Kunz and others. 
For $T=\theta$ equation (5) may be written

$$
\theta=\frac{M A I_{m}}{3 R} \text {. }
$$

Dividing equation (5) by (7), we have

But

$$
\frac{T}{\theta}=\frac{\frac{M\left(H_{0}+A I\right)}{a R}}{\frac{M A I_{m}}{3 R}}=\frac{3 H_{\bullet}}{a A I_{m}}+\frac{3}{a} \frac{I}{I_{m}} .
$$

hence

$$
I=\frac{a}{3} I_{m}
$$

Solving for $A$, we have

$$
\frac{T}{\theta}=\frac{H_{e}}{A I}+\mathbf{1}
$$

$$
A=\frac{H_{0}}{I} \frac{\theta}{T-\theta} .
$$

Knowing $A$, we can calculate $H_{m}$, the strength of the molecular field, since $H_{m}=A I$.

We can also calculate $M$, the moment of the elementary magnet, for from equation (7) we have

or

$$
\theta=\frac{M A I_{m}}{3 R}
$$

$$
M=\frac{3 R \theta}{A I_{m}} .
$$

Discussion of Results.

The curves (2) and (3), showing $\sigma$ as a function of the temperature indicate that the temperatures at which the alloys cease to be ferromagnetic is in the neighborhood of $310^{\circ} \mathrm{C}$.

Knowing $\theta$ it is possible to express $T$ as a function of the parameter $a$. Then by comparing the graph obtained with the experimental curve near the transformation point, we can calculate a value for $I_{m}$, the intensity of magnetization at absolute zero.

Equation $(5 a)$ can be written in the form

$$
I=\frac{a R T}{M A} .
$$

Dividing through by $I_{m}$, we have 
But from equation ( 7 )

$$
\frac{I}{I_{m}}=\frac{R}{M A I_{m}} a T
$$

hence

$$
\frac{R}{M A I_{m}}=\frac{\mathrm{I}}{3^{\theta}}
$$

and

$$
\frac{I}{I_{m}}=\frac{\mathrm{I}}{3 \theta} a T
$$

$$
T=\frac{3 \theta}{a} \frac{I}{I_{m}}=\frac{3^{\theta}}{a}\left(\frac{\cosh a}{\sinh a}-\frac{\mathrm{I}}{a}\right) .
$$

Putting $\theta=310^{\circ}+273^{\circ}=583^{\circ}$ and evaluating equation (12), we obtain the following values:

\begin{tabular}{c|c|c|c|c|c}
\hline$a$ & $\frac{\cosh a}{\sinh a}$ & $\frac{\sigma}{\sigma_{m}}=\frac{\cosh a}{\sinh a}-\frac{1}{a}$ & $T$ & $t$ & $74.5 \frac{\sigma}{\sigma_{m}}$ \\
\cline { 2 - 5 } 0.3 & 3.4328 & 0.0995 & 580 & 307 & 7.0 \\
0.4 & 2.6317 & 0.1317 & 577 & 304 & 9.8 \\
0.5 & 2.1639 & 0.1639 & 574 & 301 & 12.2 \\
0.6 & 1.8619 & 0.1952 & 569 & 296 & 14.5 \\
0.8 & 1.5059 & 0.2559 & 560 & 287 & 19.1 \\
1.0 & 1.3131 & 0.3131 & 548 & 275 & 23.3 \\
1.2 & 1.1995 & 0.3662 & 534 & 261 & 27.3 \\
1.6 & 1.0849 & 0.4599 & 503 & 230 & 34.3 \\
2.0 & 1.0373 & 0.5373 & 470 & 197 & 39.9 \\
3.0 & 1.0049 & 0.6716 & 392 & 119 & 50.0 \\
4.0 & 1.0007 & 0.7507 & 328 & 55 & 55.9 \\
5.0 & 1.0000 & 0.8000 & 280 & 7 & 59.6 \\
\hline \hline
\end{tabular}

Since

$$
\begin{gathered}
\frac{I}{I_{m}}=\frac{\sigma}{\sigma_{m}} \\
\sigma_{m}=\frac{\sigma}{\frac{\cosh a}{\sinh a}-\frac{I}{a}}
\end{gathered}
$$

where $\sigma_{m}$ is the value of $\sigma$ at absolute zero.

In the following table the experimental values of $\sigma$ are compared with the calculated values of $\sigma / \sigma_{m}$ for values of $t$ above $250^{\circ}$.

\begin{tabular}{c|c|c|c}
\hline$t$ & $\sigma$ Observed. & $\sigma / \sigma_{m}$ Calculated. & Value of $\sigma_{m}$. \\
\cline { 2 - 3 } 297.7 & 12.9 & .184 & 70.1 \\
297.7 & 13.5 & .184 & 73.4 \\
290.9 & 17.6 & .234 & 75.5 \\
290.9 & 16.8 & .234 & 71.8 \\
258.9 & 28.5 & .372 & 76.6 \\
\hline & & 74.5 mean \\
\hline
\end{tabular}


It is evident that if we divide the observed values of $\sigma$ by the calculated values of $\sigma / \sigma_{m}$ we can determine the value of $\sigma_{m}$. Taking the mean of several values, we get $\sigma_{m}=74.5$. If we now multiply the values of $\frac{\cosh a}{\sinh a}-\frac{1}{a}$ by 74.5 , we obtain corresponding values for $\sigma$ and $t$. Using these values we can plot a theoretical curve for $\sigma$ and the temperature. Such a curve is shown in Fig. 6.

It will be noticed that the experimental values of $\sigma$ as given by the dotted lines agree with the theoretical values for high temperatures, but at low temperatures the experimental curve bends away from the other. The same phenomenon is observed in iron, nickel and cobalt, but to a lesser degree. In the case of the magnetic alloys the bending is less (curve $B$ ) after the alloy has been chilled from a high temperature.

Since the internal or molecular field becomes negligible above the transformation temperature, the intensity of magnetization becomes proportional to the external field, and from a knowledge of the magnetic properties of the alloy in its paramagnetic state we calculate $H_{m}$ the intrinsic

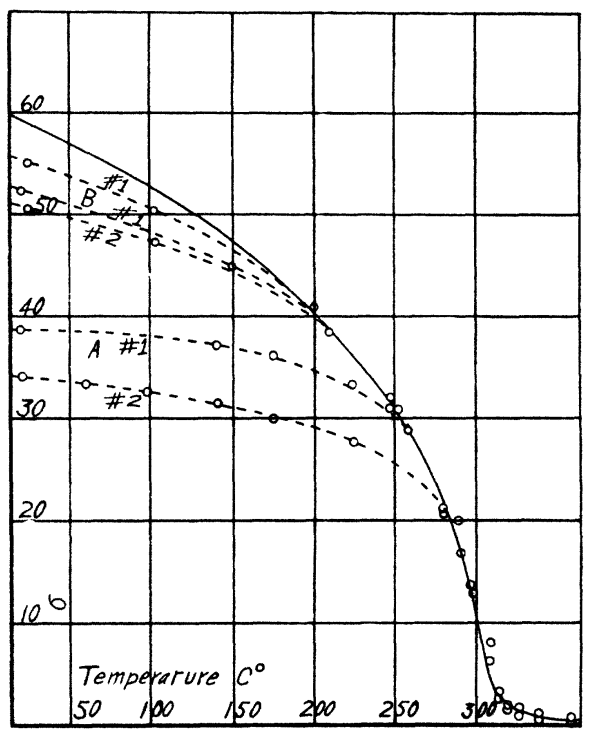

Fig. 6. molecular field and $m$ the moment of the elementary magnets.

In doing this we will first calculate $A$, the proportionality constant.

From equation (8) we have

$$
A=\frac{H_{e}}{I} \frac{\theta}{T-\theta},
$$

where $H_{c}$ is the external field and $I$ is the intensity of magnetization at temperature $T$.

Calculations of $A$ are given in the following table for various values of $T$. They give for ellipsoid No. I $A=$ 12,940; for ellipsoid No. 2 $A=$ I0,540. 
Ellipsoid No. 1.

\begin{tabular}{|c|c|c|c|c|c|}
\hline \multicolumn{3}{|c|}{ Temp. $=314.5^{\circ}}$. & \multicolumn{3}{|c|}{ Temp. $=3^{20^{\circ}}$} \\
\hline$H_{e}$ & $I$ & $K=I \mid H_{0}$ & $H_{e}$ & $I$ & $K$ \\
\hline 462 & 3.93 & .00851 & 465 & 1.98 & .00427 \\
\hline 924 & 7.42 & .00803 & 927 & 4.02 & .00434 \\
\hline 1,382 & 14.4 & .01041 & 1,390 & 6.23 & .00448 \\
\hline 1,844 & 17.9 & .00973 & 1,853 & 7.56 & .00408 \\
\hline 2,319 & 18.2 & .00788 & 2,326 & 10.25 & .00440 \\
\hline \multicolumn{2}{|c|}{ Mean .00891 } & $A=14,560$ & \multicolumn{2}{|c|}{ Mean .00439} & $A=13,270$ \\
\hline \multicolumn{3}{|c|}{ Temp. $=327.7^{\circ}$} & \multicolumn{3}{|c|}{ Temp. $=340^{\circ}$} \\
\hline$H_{e}$ & $I$ & $K$ & $H_{0}$ & $I$ & $K$ \\
\hline 464 & 1.31 & .00282 & 1,394 & 1.79 & .001284 \\
\hline 928 & 2.62 & .00282 & 2,726 & 4.15 & .001521 \\
\hline 1,392 & 3.92 & .00282 & 3,425 & 5.68 & .001658 \\
\hline 1,855 & 5.23 & .00282 & 3,695 & 7.0 & .001894 \\
\hline 2,329 & 6.54 & .00281 & & & \\
\hline \multicolumn{2}{|c|}{ Mean .00282 } & $A=11,700$ & \multicolumn{2}{|c|}{ Mean .00159} & $A=12,230$ \\
\hline
\end{tabular}

Ellipsoid No. 2.

\begin{tabular}{|c|c|c|c|c|c|}
\hline \multicolumn{3}{|c|}{ Temp. $=314.5^{\circ}$} & \multicolumn{3}{|c|}{ Temp. $=3^{20^{\circ}}$} \\
\hline$H_{e}$ & $I$ & $K=I \mid H_{c}$ & $H_{6}$ & $I$ & $K$ \\
\hline 463 & 4.61 & .00996 & 463 & 2.26 & .00489 \\
\hline 923 & 9.66 & .01046 & 926 & 5.83 & .00630 \\
\hline 1,384 & 15.14 & .01094 & 1,389 & 7.96 & .00573 \\
\hline \multirow[t]{2}{*}{1,846} & 18.92 & .01026 & 1,852 & 10.83 & .00584 \\
\hline & & & 2,326 & 12.00 & .00518 \\
\hline \multicolumn{2}{|c|}{ Mean .01040} & $A=12,460$ & \multicolumn{2}{|c|}{ Mean .00559 } & $A=10,430$ \\
\hline \multicolumn{3}{|c|}{ Temp. $=34^{\circ}$} & \multicolumn{3}{|c|}{ Temp. $=360^{\circ}$} \\
\hline$H_{e}$ & $I$ & $K$ & $H_{6}$ & $I$ & $K$ \\
\hline 1,393 & 2.74 & .001966 & 1,394 & 1.904 & .001365 \\
\hline 2,726 & 5.77 & .002115 & 2,728 & 3.38 & .001240 \\
\hline 3,425 & 6.74 & .001967 & 3,427 & 4.23 & .001233 \\
\hline 3,825 & 7.37 & .001926 & 3,827 & 4.02 & .001050 \\
\hline \multicolumn{2}{|c|}{ Mean .001993 } & $A=9,760$ & \multicolumn{2}{|c|}{ Mean .001227 } & $A=9,500$ \\
\hline \multicolumn{3}{|c|}{ Ellipsoid No. x. } & \multicolumn{3}{|c|}{ Ellipsoid No. 2.} \\
\hline \multicolumn{2}{|c|}{ Temp. } & $A$ & \multicolumn{2}{|c|}{ Temp. } & $A$ \\
\hline \multicolumn{2}{|c|}{314.5} & 14,560 & \multicolumn{2}{|c|}{314.5} & 12,460 \\
\hline \multicolumn{2}{|c|}{320.0} & 13,270 & \multicolumn{2}{|c|}{320.0} & 10,430 \\
\hline \multicolumn{2}{|c|}{327.7} & 11,700 & \multicolumn{2}{|c|}{340.0} & 9,760 \\
\hline \multicolumn{2}{|c|}{340.0} & 12,230 & \multicolumn{2}{|c|}{360.0} & 9,500 \\
\hline \multicolumn{3}{|c|}{ Mean $A=12,940$} & \multicolumn{3}{|c|}{ Mean $A=10,540$} \\
\hline
\end{tabular}


The calculation of the other quantities consists merely in substituting in the equations already obtained. The work is summarized as follows:

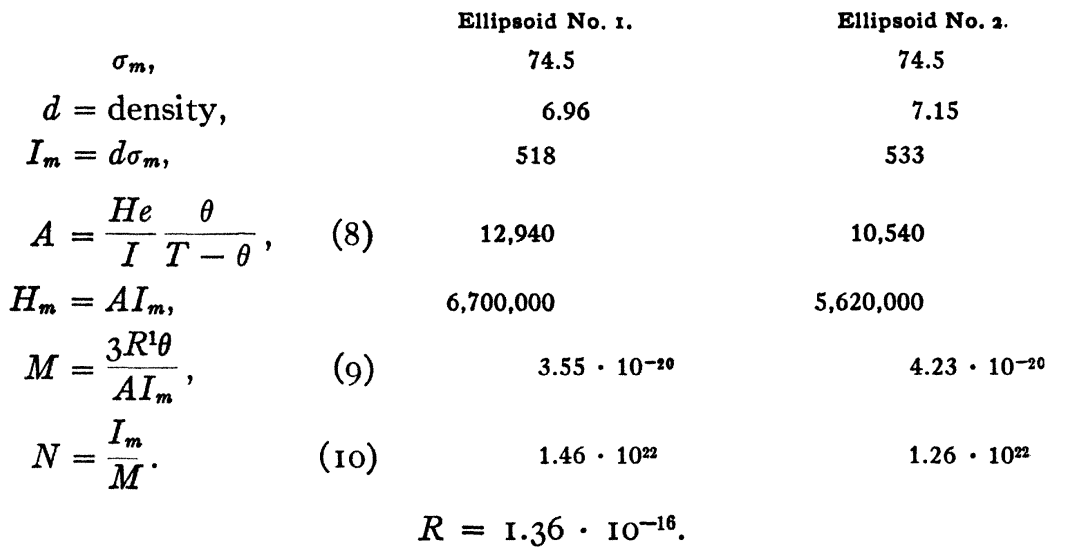

For comparison, the constants obtained for iron and nickel by $\mathrm{Kunz}^{2}$ and for cobalt by Stifler ${ }^{3}$ are given in the following table:

\begin{tabular}{|c|c|c|c|c|c|c|}
\hline & $I_{m}$ & $\theta$ & $A$ & $H_{m}$ & $M \times 10^{-20}$. & $N \times 10^{22}$ \\
\hline Iron. & 2,120 & $756^{\circ} \mathrm{C}$. & 3,850 & $6,560,000$ & 5.15 & 4.12 \\
\hline Cobalt. & 1,435 & 1,075 & 6,180 & $8,870,000$ & 6.21 & 2.31 \\
\hline Nickel & 570 & 376 & 12,700 & $6,350,000$ & 3.65 & 1.56 \\
\hline Alloy No. 1. & 518 & 310 & 12,940 & $6,700,000$ & 3.55 & 1.46 \\
\hline Alloy No. 2. & 533 & 310 & 10,540 & $5,620,000$ & 4.23 & 1.26 \\
\hline
\end{tabular}

If we assume that there is one atom of manganese in the magnetic molecule then for alloy number one we have the relation

$$
m_{H}=\frac{d}{w n},
$$

where $m_{H}=$ weight of a hydrogen atom.

$w=55=$ atomic weight of manganese.

$n=1.46 \cdot 10^{22}$ the number of magnetic molecules per cu. $\mathrm{cm}$.

$d=$ density of alloy $\times$ per cent. of manganese in alloy.

$=6.96 \cdot 0.185$.

$$
m_{H}=\frac{6.96 \cdot 0.185}{55 \cdot 1.46} \mathrm{IO}^{22}=\mathrm{I} .60 \cdot \mathrm{IO}^{-24}
$$

${ }^{1} R$ is the universal gas constant, and the value to be used is that corresponding to one molecule.

2 Phys. Rev., Vol. 30, p. 259 (r9io).

8 Puys. Rev., Vol. 33, p. 268 (rgri). 
This value of $m_{B}$ agrees almost exactly with $\mathrm{I} .6 \mathrm{I} \cdot 1 \mathrm{IO}^{-24}$, the value obtained by Rutherford. From alloy number two we get $m_{H}=2.12 \cdot 10^{-24}$, which does not agree very closely.

If $e$ is the elementary charge of the hydrogen atom and $\alpha_{H}$ is the chemical equivalent of hydrogen, then $e=m_{H} / \alpha_{H}$. Using the values of $m_{H}$ obtained above we get from alloy number one $e=1.54 \cdot 10^{-20}$ and from alloy number two $e=2.04 \cdot 10^{-20}$.

We could have obtained, however, the same values by assuming that the magnetic molecule was composed of one atom of manganese and one atom of aluminium, or one atom of manganese and one atom of copper. Since a molecule must contain more than one atom, it is quite probable that the magnetic molecule is a composite molecule containing one atom of mangenese and one atom either of copper or of aluminium. This hypothesis would also account for the increase in the intensity of magnetism after chilling from a high temperature, as shown in Fig. 6. The chilling prevents one of the metals from crystallizing out and thus prevents a decrease in the number of molecular magnets.

The author hopes to continue the investigation and to determine definitely the number and kind of atoms in the elementary magnet, and also to study the effect of the percentage of copper upon the transformation temperature. These results will be important in proving that the magnetic properties are due to the manganese.

\section{SUMMARY.}

The chief results of this investigation may be summarized as follows:

I. The temperature of magnetic transformation from the ferromagnetic to the paramagnetic state was established at $310^{\circ} \mathrm{C}$. for the alloys containing 62 per cent. copper.

2. The curve giving $\sigma$ as a function of the temperature has been shown to agree with the theoretical curve above $200^{\circ}$.

3. Chilling from near the melting point causes the experimental curve to follow the theoretical curve to a lower temperature than before.

4. The nature of the molecular field was found to be of the same order of magnitude as nickel, all the constants $I_{m}, H_{m}, M$ and $H$, being of approximately the same value.

5. The results, while not extensive enough to determine the number and kind of atoms in the elementary magnet, are sufficient to show that the alloys obey the laws of ferro-magnetism, as derived by the present molecular theory.

6. The fundamental equation (I), on which the present theory of 
magnetism is based, has been derived mathematically; thereby, making the former analogy to the gas theory unnecessary.

The writer takes pleasure in acknowledging his indebtedness to Professor Jakob Kunz for his general supervision of the work, and for many valuable suggestions.

LABORATORY OF PHYSICS,

UNIVERSITY OF ILLINOIS,

April 25, I9I2. 